\title{
An Operating Precision Analysis Method Considering Multiple Error Sources of Serial Robots
}

\author{
Cong Wei ${ }^{a}$, Qingxuan Jia and Gang Chen \\ Beijing University of Posts and Telecommunications, Beijing, China
}

\begin{abstract}
In order to solve the problem of operating precision analysis considering multiple error sources of serial robots, an operating precision analysis method combined Monte Carlo algorithm with pose error model of robot endeffector is proposed. Firstly, the pose error model of robot end-effector is built based on the MCPC method. Then, the error sources which have a main influence on the operating precision of robot end-effector are analysed in detail. At last, the Monte Carlo algorithm is used to analyse the error probabilistic characteristics of robot end-effector considering multiple error sources, which can validate the precision design of mechanical arms and provide theory basis to distribute the error limits of error sources reasonably.
\end{abstract}

\section{Introduction}

The operating precision is one of the important indicators of evaluating the usability of robot, and the level of operating precision will directly influence the effect of task execution. The error sources which affect the operating precision of the end-effector are various, such as manufacture and install error, joint clearance, flexibility of connecting rod and joint, working environment and so on[1]. The operating precision analysis of the end effector can be judged if it meets the requirement of precision and provide theory basis to distribute the error limits of error sources reasonably. There are abundant researches on the precision of the end-effector both at home and abroad in the past years. Jeong Kima[2] used the improved first-order secondmoment method to analyse the effects of the joint clearance on the precision of end effector. Meng Xianju[3] used geometric model to study the effects of joint motion error on the precision of end effector. Tian Li[4] established the mathematical model of the flexible joint, which is used to calculate the pose error of the robot endeffector. Zhang Zhiweng[5] researched the effects of connecting rod deformation on the precision of endeffector under their own gravity and load. In conclusion, although many scholars had studied the operating precision of serial robot, the studies which considering multiple error sources at the same time for operating precision are still rarely.

In this paper, an operating precision analysis method is proposed which combines Monte Carlo algorithm with pose error model of robot end-effector. Section 2 is about the establishment of pose error model; Section 3 is about the analysis of error sources; Section 4 is about the

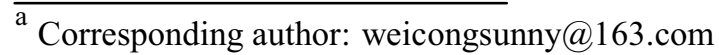

probability analysis of operating precision; the conclusion is in Section 5.

\section{Pose Error Model}

\subsection{Kinematic Model}

The most classical kinematic modeling method is based on D-H method proposed by Denavit and HartenBerg[6]. However, when adjacent joint axes of manipulator are parallel, it will occur large position offset on common normal line because of the error of parallelism. Therefore, in this paper, the kinematic model is built by MCPC method, which can ensure the integrity and continuity of the model. It uses four parameters $\alpha, \beta, x, y$ to describe the transformation relation between connecting rod coordinates, Where $\alpha, \beta$ represent the angle rotating on $x$ axis and the angle rotating on $y$ axis when coordinate $i$ transforming to coordinate $i+1$, respectively; $x$ represents the translational distance between the origin of coordinate $i$ and origin of coordinate $i+1$ in the direction of $x$ axis; $y$ represents the translational distance between the origin of coordinate $i$ and origin of coordinate $i+1$ in the direction of $y$ axis. And six parameters $\alpha, \beta, x, y, \gamma, z$ is used to describe the transformation relation between terminate coordinate and tool coordinate, Where $\gamma$ represents the angle rotating on $z$ axis; $z$ represents the translational distance between the origin of coordinate $i$ and origin of coordinate $i+1$ in the direction of $z$ axis [7].

The transform matrix between intermediate connecting rod coordinates is:

$$
\boldsymbol{T}_{i}=\boldsymbol{Q}_{i} \operatorname{Rot}\left(x, \alpha_{i}\right) \operatorname{Rot}\left(y, \beta_{i}\right) \operatorname{Trans}\left(x_{i}, y_{i}, 0\right)
$$


And the transform matrix between terminate coordinate and tool coordinate is:

$$
\boldsymbol{T}_{n}=\boldsymbol{Q}_{n} \operatorname{Rot}\left(x, \alpha_{n}\right) \operatorname{Rot}\left(y, \beta_{n}\right) \operatorname{Rot}\left(z, \gamma_{n}\right) \operatorname{Trans}\left(x_{n}, y_{n}, z_{n}\right)
$$

At last, the kinematic model is derived as follows:

$$
\boldsymbol{T}=\boldsymbol{T}_{0} \cdot \boldsymbol{T}_{1} \cdots \boldsymbol{T}_{n-1} \cdot \boldsymbol{T}_{n}
$$

\subsection{Pose Error Model}

$d \boldsymbol{T}_{i}$ is defined as the difference between the real pose of the end-effector and the desired one:

$$
d \boldsymbol{T}_{i}=\boldsymbol{T}_{i}^{\prime}-\boldsymbol{T}_{i}=\boldsymbol{T}_{i} \cdot{ }^{T} \boldsymbol{\Delta} \quad(i=0,1, \cdots, n-1)
$$

Where $\boldsymbol{T}_{i}, \boldsymbol{T}_{i}^{\prime}$ represent the desired transform matrix and the real transform matrix between coordinate $i$ and coordinate $i+1$, respectively.

Then, the pose error matrix ${ }^{T} \boldsymbol{\Delta}_{i}$ can be derived as follows:

$$
{ }^{T} \boldsymbol{\Delta}_{i}=\boldsymbol{T}_{i}^{-1} \cdot\left(\boldsymbol{T}_{i}^{\prime}-\boldsymbol{T}_{i}\right)=\left[\begin{array}{cccc}
0 & -\delta_{i z} & \delta_{i y} & d_{i x} \\
\delta_{i z} & 0 & -\delta_{i x} & d_{i y} \\
-\delta_{i y} & \delta_{i x} & 0 & d_{i z} \\
0 & 0 & 0 & 0
\end{array}\right]
$$

Because there are two extra parameters $\gamma, z$ to describe the transformation relation between terminate coordinate and tool coordinate, the pose error model need aim at intermediate connecting rod coordinate and terminate coordinate, respectively.

For the intermediate connecting rod coordinates, $d \boldsymbol{T}_{i}$ is mainly related to the linkage parameters $\alpha_{i}, \beta_{i}, x_{i}, y_{i}$ and the joint parameter $\theta_{i}$ :

$$
\begin{aligned}
& d \boldsymbol{T}_{i}=\frac{\partial \boldsymbol{T}_{i}}{\partial \theta_{i}} \Delta \theta_{i}+\frac{\partial \boldsymbol{T}_{i}}{\partial \alpha_{i}} \Delta \alpha_{i}+\frac{\partial \boldsymbol{T}}{\partial \beta_{i}} \Delta \beta_{i}+\frac{\partial \boldsymbol{T}_{i}}{\partial x_{i}} \Delta x_{i}+\frac{\partial \boldsymbol{T}_{i}}{\partial y_{i}} \Delta y_{i} \\
& \frac{\partial \boldsymbol{T}_{i}}{\partial \theta_{i}}=\boldsymbol{T}_{i} \cdot \boldsymbol{Q}_{\theta i}, \frac{\partial \boldsymbol{T}_{i}}{\partial \alpha_{i}}=\boldsymbol{T}_{i} \cdot \boldsymbol{Q}_{\alpha i}, \frac{\partial \boldsymbol{T}_{i}}{\partial \beta_{i}}=\boldsymbol{T}_{i} \cdot \boldsymbol{Q}_{\beta i}, \frac{\partial \boldsymbol{T}_{i}}{\partial x_{i}}=\boldsymbol{T}_{i} \cdot \boldsymbol{Q}_{x i},
\end{aligned}
$$

$\frac{\partial \boldsymbol{T}_{i}}{\partial y_{i}}=\boldsymbol{T}_{i} \cdot \boldsymbol{Q}_{y i}$, Then ${ }^{T} \boldsymbol{\Delta}_{i}$ can be derived based on Eq. (4), Eq. (5) and Eq. (6):

$$
{ }^{T} \boldsymbol{\Delta}=\boldsymbol{Q}_{\theta i} \Delta \theta_{i}+\boldsymbol{Q}_{\alpha i} \Delta \alpha_{i}+\boldsymbol{Q}_{\beta i} \Delta \beta_{i}+\boldsymbol{Q}_{x i} \Delta x_{i}+\boldsymbol{Q}_{y i} \Delta y_{i}
$$

$\boldsymbol{d}_{i}$ is defined as positional error and $\boldsymbol{\delta}_{i}$ is defined as attitude error, then the pose error vector $\boldsymbol{D}_{i}$ can be gotten as follows:

$$
\boldsymbol{D}_{i}=\left[\begin{array}{l}
\boldsymbol{d}_{i} \\
\boldsymbol{\delta}_{i}
\end{array}\right]=\left[\begin{array}{ccccc}
\boldsymbol{k}_{i}^{1} & \boldsymbol{k}_{i}^{2} & \boldsymbol{k}_{i}^{3} & \boldsymbol{k}_{i}^{4} & \boldsymbol{k}_{i}^{5} \\
\boldsymbol{k}_{i}^{6} & \boldsymbol{k}_{i}^{7} & \boldsymbol{k}_{i}^{8} & \boldsymbol{0}_{3 \times 1} & \boldsymbol{0}_{3 \times 1}
\end{array}\right]\left[\begin{array}{c}
\Delta \theta_{i} \\
\Delta \alpha_{i} \\
\Delta \beta_{i} \\
\Delta x_{i} \\
\Delta y_{i}
\end{array}\right]
$$

For the terminate coordinate, $d \boldsymbol{T}_{n}$ is mainly related to the joint parameter $\theta_{n}$ and the linkage parameters $\alpha_{n}, \beta_{n}, x_{n}, y_{n}, \gamma_{n}, z_{n}$.

$$
\begin{aligned}
d \boldsymbol{T}_{n}= & \frac{\partial \boldsymbol{T}_{n}}{\partial \theta_{n}} \Delta \theta_{n}+\frac{\partial \boldsymbol{T}_{n}}{\partial \alpha_{n}} \Delta \alpha_{n}+\frac{\partial \boldsymbol{T}_{n}}{\partial \beta_{n}} \Delta \beta_{n}+\frac{\partial \boldsymbol{T}_{n}}{\partial \gamma_{n}} \Delta \gamma_{n} \\
& +\frac{\partial \boldsymbol{T}_{n}}{\partial x_{n}} \Delta x_{n}+\frac{\partial \boldsymbol{T}_{n}}{\partial y_{n}} \Delta y_{n}+\frac{\partial \boldsymbol{T}_{n}}{\partial z_{n}} \Delta z_{n}
\end{aligned}
$$

$$
\frac{\partial \boldsymbol{T}_{n}}{\partial \theta_{n}}=\boldsymbol{T}_{n} \cdot \boldsymbol{Q}_{\theta n}, \frac{\partial \boldsymbol{T}_{n}}{\partial \alpha_{n}}=\boldsymbol{T}_{n} \cdot \boldsymbol{Q}_{\alpha n}, \frac{\partial \boldsymbol{T}_{n}}{\partial \beta_{n}}=\boldsymbol{T}_{n} \cdot \boldsymbol{Q}_{\beta n}, \frac{\partial \boldsymbol{T}_{n}}{\partial \gamma_{n}}=\boldsymbol{T}_{n} \cdot \boldsymbol{Q}_{\gamma n}
$$

$\frac{\partial \boldsymbol{T}_{n}}{\partial x_{n}}=\boldsymbol{T}_{n} \cdot \boldsymbol{Q}_{x n}, \frac{\partial \boldsymbol{T}_{n}}{\partial y_{n}}=\boldsymbol{T}_{n} \cdot \boldsymbol{Q}_{y n}$, Then ${ }^{T} \boldsymbol{\Delta}_{n}$ can be derived based on Eq. (4), Eq. (5) and Eq. (9):

$$
\begin{aligned}
{ }^{T} \boldsymbol{\Delta}_{n}= & \boldsymbol{Q}_{\theta n} \Delta \theta_{n}+\boldsymbol{Q}_{\alpha n} \Delta \alpha_{n}+\boldsymbol{Q}_{\beta n} \Delta \beta_{n}+\boldsymbol{Q}_{\gamma n} \Delta \gamma_{n} \\
& +\boldsymbol{Q}_{x n} \Delta x_{n}+\boldsymbol{Q}_{y n} \Delta y_{n}+\boldsymbol{Q}_{z n} \Delta z_{n}
\end{aligned}
$$

$\boldsymbol{d}_{n}$ is defined as positional error and $\boldsymbol{\delta}_{n}$ is defined as attitude error, then the pose error vector $\boldsymbol{D}_{n}$ can be gotten as follows:

$$
\boldsymbol{D}_{n}=\left[\begin{array}{c}
\boldsymbol{d}_{n} \\
\boldsymbol{\delta}_{n}
\end{array}\right]=\left[\begin{array}{lllllll}
\boldsymbol{k}_{n}^{1} & \boldsymbol{k}_{n}^{2} & \boldsymbol{k}_{n}^{3} & \boldsymbol{k}_{n}^{9} & \boldsymbol{k}_{n}^{4} & \boldsymbol{k}_{n}^{5} & \boldsymbol{k}_{n}^{10} \\
\boldsymbol{k}_{n}^{6} & \boldsymbol{k}_{n}^{7} & \boldsymbol{k}_{n}^{8} & \boldsymbol{k}_{n}^{11} & \boldsymbol{0}_{3 \times 1} & \boldsymbol{0}_{3 \times 1} & \boldsymbol{0}_{3 \times 1}
\end{array}\right]\left[\begin{array}{c}
\Delta \theta_{n} \\
\Delta \alpha_{n} \\
\Delta \beta_{n} \\
\Delta \gamma_{n} \\
\Delta x_{n} \\
\Delta y_{n} \\
\Delta z_{n}
\end{array}\right]
$$

$\boldsymbol{T}, \boldsymbol{T}^{\prime}$ are defined as the desired transform matrix and the real transform matrix between terminate coordinate and inertial coordinate, respectively:

$$
\begin{aligned}
\boldsymbol{T}^{\prime} & =\boldsymbol{T}+d \boldsymbol{T}=\prod_{i=0}^{n}\left(\boldsymbol{T}_{i}+d \boldsymbol{T}_{i}\right) \\
& =\boldsymbol{T}+\sum_{i=0}^{n} \boldsymbol{T}_{0} \boldsymbol{T}_{1} \boldsymbol{T}_{2} \cdots \boldsymbol{T}_{i-1} \cdot d \boldsymbol{T}_{i} \cdot \boldsymbol{T}_{i+1} \cdots \boldsymbol{T}_{n}
\end{aligned}
$$

Then, $d \boldsymbol{T}$ is gotten as follows:

$$
d \boldsymbol{T}=\boldsymbol{T} \cdot{ }^{T} \boldsymbol{\Delta}=\sum_{i=0}^{n} \boldsymbol{T} \cdot\left(\boldsymbol{U}_{i+1}\right)^{-1} \cdot{ }^{T} \boldsymbol{\Delta} \cdot \boldsymbol{U}_{i+1}
$$

Where, $\boldsymbol{U}_{n+1}=\boldsymbol{E}_{4}, \quad \boldsymbol{E}_{4}$ is the identity matrix, $\boldsymbol{U}_{i}=\boldsymbol{T}_{i} \boldsymbol{T}_{i+1} \cdots \boldsymbol{T}_{n}=\left[\begin{array}{rrrr}\boldsymbol{n}_{i}^{u} & \boldsymbol{o}_{i}^{u} & \boldsymbol{a}_{i}^{u} & \boldsymbol{p}_{i}^{u} \\ 0 & 0 & 0 & 1\end{array}\right]=\left[\begin{array}{cc}\boldsymbol{R}_{i}^{u} & \boldsymbol{p}_{i}^{u} \\ \boldsymbol{0} & 1\end{array}\right]$.

${ }^{T} \boldsymbol{\Delta}$ is derived as follows:

$$
{ }^{T} \boldsymbol{\Delta}=\sum_{i=0}^{n}\left(\boldsymbol{U}_{i+1}\right)^{-1} \cdot{ }^{T} \boldsymbol{\Delta} \cdot \boldsymbol{U}_{i+1}=\left[\begin{array}{cccc}
0 & -\delta_{z} & \delta_{y} & d_{x} \\
\delta_{z} & 0 & -\delta_{x} & d_{y} \\
-\delta_{y} & \delta_{x} & 0 & d_{z} \\
0 & 0 & 0 & 0
\end{array}\right]
$$

The pose error vector $\boldsymbol{D}_{e}$ of the end-effector in the terminate coordinate can be gotten: 


$$
\boldsymbol{D}_{e}=\left[\begin{array}{l}
d_{x} \\
d_{y} \\
d_{z} \\
\delta_{x} \\
\delta_{y} \\
\delta_{z}
\end{array}\right]=\sum_{i=0}^{n}\left(\left[\begin{array}{cc}
\left(\boldsymbol{n}_{i+1}^{u}\right)^{T} & \left(\boldsymbol{p}_{i+1}^{u} \times \boldsymbol{n}_{i+1}^{u}\right)^{T} \\
\left(\boldsymbol{o}_{i+1}^{u}\right)^{T} & \left(\boldsymbol{p}_{i+1}^{u} \times \boldsymbol{o}_{i+1}^{u}\right)^{T} \\
\left(\boldsymbol{a}_{i+1}^{u}\right)^{T} & \left(\boldsymbol{p}_{i+1}^{u} \times \boldsymbol{a}_{i+1}^{u}\right)^{T} \\
\boldsymbol{0}_{1 \times 3} & \left(\boldsymbol{n}_{i+1}^{u}\right)^{T} \\
\boldsymbol{0}_{1 \times 3} & \left(\boldsymbol{o}_{i+1}^{u}\right)^{T} \\
\boldsymbol{0}_{1 \times 3} & \left(\boldsymbol{a}_{i+1}^{u}\right)^{T}
\end{array}\right]\left[\begin{array}{c}
\boldsymbol{d}_{i} \\
\boldsymbol{\delta}_{i}
\end{array}\right]\right)
$$

The Eq. (15) is deduced based on Eq. (8) and Eq. (11):

$$
\boldsymbol{D}_{e}=\left[\begin{array}{ccccccc}
\boldsymbol{M}_{1} & \boldsymbol{M}_{2} & \boldsymbol{M}_{3} & \boldsymbol{k}_{n}^{9} & \boldsymbol{M}_{4} & \boldsymbol{M}_{5} & \boldsymbol{k}_{n}^{10} \\
\boldsymbol{M}_{6} & \boldsymbol{M}_{7} & \boldsymbol{M}_{8} & \boldsymbol{k}_{n}^{11} & \boldsymbol{O}_{3 \times(n+1)} & \boldsymbol{0}_{3 \times(n+1)} & \boldsymbol{0}_{3 \times 1}
\end{array}\right]\left[\begin{array}{c}
\boldsymbol{\Delta} \boldsymbol{\theta} \\
\boldsymbol{\Delta} \boldsymbol{\alpha} \\
\boldsymbol{\beta} \boldsymbol{\beta} \\
\Delta \gamma_{n} \\
\boldsymbol{\Delta x} \\
\boldsymbol{\Delta y} \\
\Delta z_{n}
\end{array}\right]
$$

$$
=J_{E} \cdot \Delta \Omega
$$

The Eq. (16) shows the mapping relation between linkage errors, joint errors and pose error in the terminate coordinate. Furthermore, the pose error model of the endeffector $\boldsymbol{D}_{e}^{\prime}$ in the inertial coordinate can be gotten:

$$
\boldsymbol{D}_{e}^{\prime}=\boldsymbol{T}_{s} \cdot \boldsymbol{J}_{E} \cdot \Delta \boldsymbol{\Omega}=\boldsymbol{J}_{I} \cdot \Delta \boldsymbol{\Omega}
$$

\section{Analysis of Error Sources}

In the part 2, the mapping relation between linkage errors, joint errors and pose error can be gotten in the inertial coordinate. It will be conducive to the operating precision analysis if all the error sources are transformed into linkage errors and joint errors.

\subsection{Error Analysis of Manufacture and Install Error}

The manufacture error mainly shows in the length error $\Delta L_{i}$ of connecting rod, which causes the linkage errors $\Delta x, \Delta y, \Delta z$.

The install error mainly shows in the joint axial deviation, which can be seen in Fig.1.

$$
\begin{aligned}
& \boldsymbol{d}=\left[d_{x}, d_{y}, 0\right]=\overrightarrow{O O^{\prime}}=\overrightarrow{O A}+\overrightarrow{A O^{\prime}}=\overrightarrow{O A}+\overrightarrow{A B} \frac{\left|A O^{\prime}\right|}{|A B|} \\
& \boldsymbol{k}=\left[k_{x}, k_{y}, 0\right]=\left[-y_{A E}, x_{A E}, 0\right] /|\overrightarrow{A E}|, \delta \varphi \approx \tan \delta \varphi=\frac{B C}{A C}
\end{aligned}
$$

Where, $\boldsymbol{d}$ denotes the differential motion of the joint axis; $\boldsymbol{k}$ denotes the rotational axis; $\delta \varphi$ denotes the differential angle rotating on $\boldsymbol{k}$ axis; the line $A B$ denotes the real joint axis.

Then, the pose error of corresponding coordinate can be gotten as follows:

$$
\boldsymbol{D}_{k}=\left[k_{x} \cdot \delta \varphi, k_{y} \cdot \delta \varphi, 0, d_{x}, d_{y}, 0\right]^{T}
$$

The linkage errors $\Delta \boldsymbol{\Omega}_{k}$ can be deduced based on Eq. (15), Eq. (16) and Eq. (19):

$$
\Delta \boldsymbol{\Omega}_{k}=\boldsymbol{J}_{k}^{-1} \cdot \boldsymbol{D}_{k}
$$

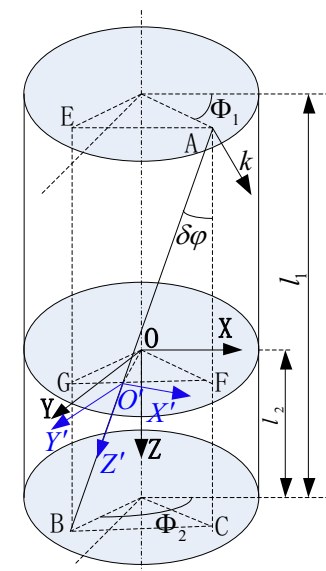

Figure 1. The joint axial deviation caused by install error.

\subsection{Error Analysis of Flexibility of Connecting Rod}

The force of connecting rod $i$ is analyzed, which can be seen in Fig.2. Set the length of connecting rod as $l$. The left of the connecting rod is fixed, and force $\boldsymbol{F}=\left(F_{x}, F_{y}, F_{z}\right)^{T}$, torque $\boldsymbol{M}=\left(M_{x}, M_{y}, M_{z}\right)^{T}$ are applied on the right of the connecting rod. The uniform load $\boldsymbol{q}$ is created by the connecting rod's own weight. Because of the effect of forces and torque, the right of the connecting rod will move from $\mathrm{A} 1$ to $\mathrm{A} 2$, and the pose error of corresponding coordinate can be gotten as follows:

$$
\boldsymbol{D}_{i}=\left[\begin{array}{llllll}
\Delta l & w_{y} & w_{z} & \Delta \varphi & \theta_{z} & \theta_{y}
\end{array}\right]^{T}
$$

Where, $\Delta l$ represents the tension or compression deformation; $w_{y}$ represents the bending deflection in the direction of $y$ axis; $w_{z}$ represents the bending deflection in the direction of $z$ axis; $\Delta \varphi$ represents the twist; $\theta_{z}$ represents the angle rotating on $z$ axis; $\theta_{y}$ represents the angle rotating on $y$ axis.

The linkage errors $\Delta \Omega$ can be deduced based on Eq. (15), Eq. (16) and Eq. (21):

$$
\Delta \boldsymbol{\Omega}_{i}=\boldsymbol{J}_{i}^{-1} \cdot \boldsymbol{D}_{i}
$$

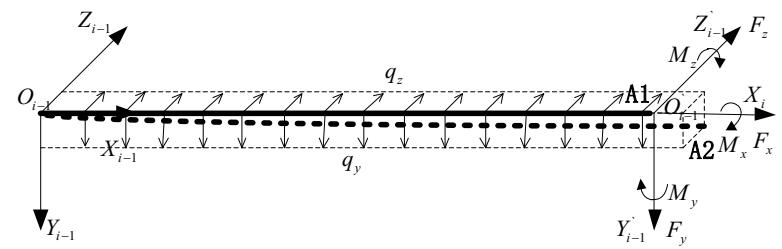

Figure 2. The deformation of connecting rod.

\subsection{Error Analysis of Flexibility of Joint}

The flexible joint of manipulator is simplified to a torsional spring, where flexible deformation of the joint is proportional to the torque of joint [8]: 


$$
d \theta_{i}=C_{i} \cdot \tau_{i}
$$

Based on above analysis, the error sources can be transformed into linkage errors or joint errors. When the manipulator has a high stiffness, these variable errors can be linearly added. Then the pose error of the end-effector in the inertial coordinate can be gotten through Eq. (17) with multiple error sources affecting the operating precision of the end-effector.

\section{Probability Analysis of Operating Precision}

The value of error sources has randomness. It's very low probability that each value of error source has an extreme. If set the extreme of error source as the robot design indicators, it will increase the cost of manufacture. Therefore, the probability analysis of operating precision is needed. In this paper, the probability analysis method of operating precision is proposed based on Monte Carlo algorithm, and a four link serial robot is considered. The 3-D shape of 4-DOF serial robot is shown in Fig.3. The MCPC Coordinates of 4-DOF serial robot is shown in Fig.4. The MCPC parameters are shown in Tab.1.

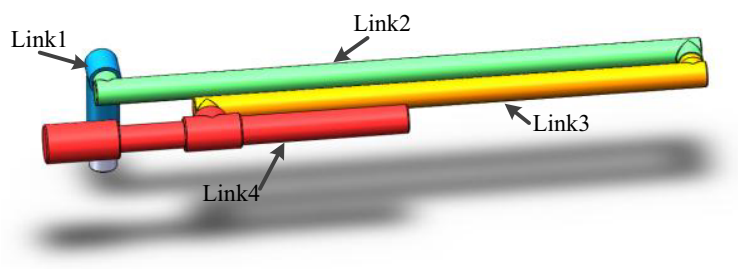

Figure 3. The 3-D shape of 4-DOF serial robot.

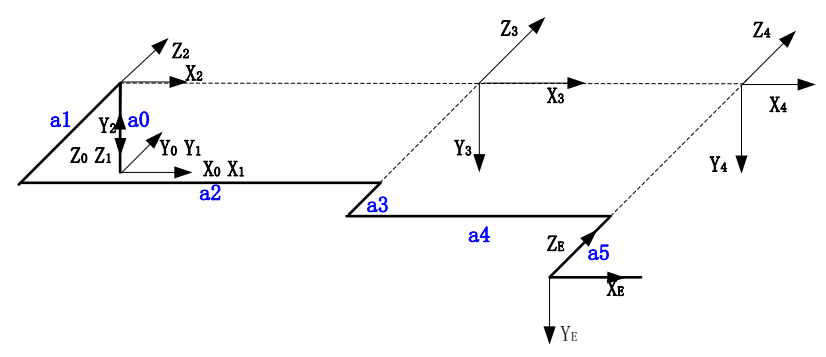

Figure 4. The MCPC Coordinates of 4-DOF serial robot.

Table 1. The MCPC parameters of 4-DOF serial robot.

\begin{tabular}{|c|c|c|c|c|c|c|}
\hline & $\alpha_{i}$ & $\beta_{i}$ & $\gamma_{i}$ & $x_{i}(\mathrm{~mm})$ & $y_{i}(\mathrm{~mm})$ & $z_{i}(\mathrm{~mm})$ \\
\hline 0 & 0 & 0 & $\backslash$ & 0 & 0 & $\backslash$ \\
\hline 1 & $-90^{0}$ & 0 & $\backslash$ & 0 & $-a_{1}$ & $\backslash$ \\
\hline 2 & 0 & 0 & $\backslash$ & $a_{2}$ & 0 & $\backslash$ \\
\hline 3 & 0 & 0 & $\backslash$ & $a_{4}$ & 0 & $\backslash$ \\
\hline 4 & 0 & 0 & 0 & 0 & 0 & $-a_{1}-a_{3}-a_{5}$ \\
\hline
\end{tabular}

Where, $a_{0}=96, a_{1}=85, a_{2}=1970, a_{3}=96, a_{4}=1770$, $a_{5}=93$; the length error of $a_{0}, a_{1}, a_{3}, a_{5}$ is $\pm 0.05 \mathrm{~mm}$; the length error of $a_{2}, a_{4}$ is $\pm 0.25 \mathrm{~mm}$; the install error of joint axial is $0.1 \mathrm{~mm}$; the quality of Link 2 is $1.4 \mathrm{~kg}$, the torsional rigidity of Link2 is $G I_{p 2}=4000 \mathrm{Nm} / \mathrm{rad}$, and the bending rigidity of Link2 is $E I_{2}=8000 \mathrm{Nm} / \mathrm{rad}$; the quality of Link3 is $1.4 \mathrm{~kg}$, the torsional rigidity of Link3 is $G I_{p 3}=1000 \mathrm{Nm} / \mathrm{rad}$, and the bending rigidity of Link3 is $E I_{3}=8000 \mathrm{Nm} / \mathrm{rad}$; the quality of Link4 is $3.3 \mathrm{~kg}$; the quality of the load is $1 \mathrm{~kg}$.

The probability analysis steps of operating precision can be divided into:

1) Obtain the probability distribution of each error source;

2) Generate a set of error values of error sources randomly, and transform them into linkage errors and joint errors.

3 ) Get the pose error of the end-effector through Eq. (14) using the variable errors generated in step 2;

4) Perform the steps above $N$ times, and then $N$ groups value of pose error can be gotten. When the value of $N$ is big enough, the probability distribution of the pose error of the end-effector can be gotten.

The pose error of robot end-effector is not only concerned with the value of error sources, but also concerned with the configuration of manipulator. Here, the joint angles are $\left[-90^{\circ} 3^{0} 88^{0}-91^{0}\right], N=2000$, then do the probability analysis of operating precision by the steps mentioned above. The probability distribution of the pose error of the end-effector is shown in Fig.5 and Fig.6.

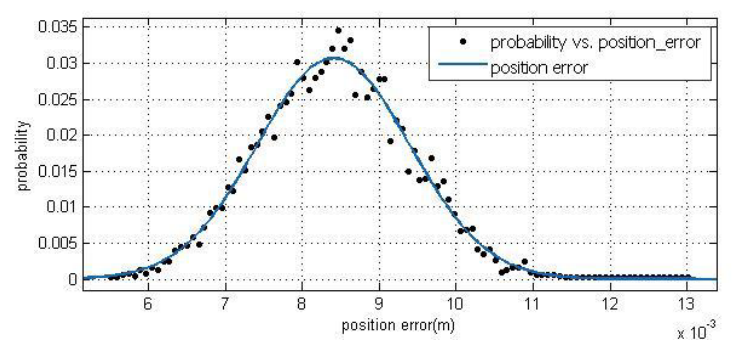

Figure 5. The position error of the end-effector.

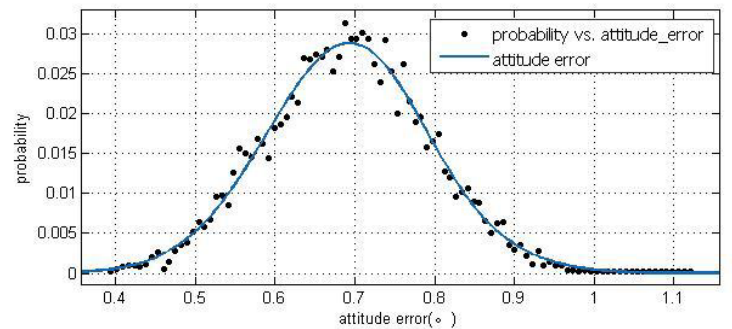

Figure 6. The attitude error of the end-effector.

As shown in Fig.5 and Fig.6, we can get the minimum and maximum of the position error, the minimum and maximum of the attitude error, and the value of the highest probability. It can be used to estimate the operating precision of the end-effector intuitively, and verify whether the design of the manipulator meets the precision requirement. What's more, it can provide theory basis to distribute the error limits of error sources reasonably. 


\section{Conclusion}

An operating precision analysis method is proposed which combines Monte Carlo algorithm with pose error model of robot end-effector. Firstly, the pose error model of robot end-effector is built based on MCPC method, which represents the mapping relation between linkage errors, joint errors and pose error in the terminate coordinate. Then the main error sources which influence the operating precision of robot end-effector are analyzed in detail and transformed into linkage errors and joint errors. At last, we get the probability distribution of the pose error of the end-effector. From the probability analysis result of operating precision we can verify whether the design of the manipulator meets the precision requirement, and provide theory basis to distribute error limits of error sources reasonably.

\section{ACKNOWLEDGMENT}

This research is supported by the National Natural Science Foundation of China (61403038) and the National Natural Science Foundation of China (61573058)

\section{References}

1. Jiao Guotai, Analysis and synthesis of robot pose errors, Post-doctor work report of Beijing University of Technology. (2002)

2. Jeong Kima, Woo-Jin Song, Beom-Soo Kang, Stochastic approach to kinematic relia-bility of openloop mechanism with dimensional tolerance. Applied Mathematical Model-ing 34(5): 1225-1237 (2009)

3. Meng XianJu, SHI ZhongXiu, ZHAN MinJing, ZHANG Ce, Analysis on probabilistic characteristics of motion error in link mechanism. Journal of Machine Design 20(1): 47-52 (2003)

4. Li Tian, Research on Flexibile Joint Robot and Its Kinematic Calibration and Vibration Suppression Reserch. Harbin Institute of Technology (2012)

5. Zhang Zhiwen, Gan Fangjian, Robot Motion Error Based on Links's Gravity. Mechanical Research \& Application 01(19): 73-77 (2006)

6. Cai Zixing, Robotics. Beijing: Tsinghua University Press (2000)

7. Hanqi Zhuang, Luke K. Wang, Zvi S. Roth, ErrorModel-Based Robot Calibration Using A Modifued Cpc Model. Robotics and Computer-Integrated Manufacturing 10(4): 287-299 (1993)

8. Spong M W, Robot dyamics and control. NewYork: John wiley\&Sons (1989) 